\title{
Notas sobre a composição arbóreo-arbustiva de uma fisionomia das savanas de Roraima, Amazônia Brasileira
}

\author{
Reinaldo Imbrozio Barbosa ${ }^{1,4}$, Sebastião Pereira do Nascimento ${ }^{2}$, Paulo Atlântico Figueiredo de Amorim ${ }^{3} \mathrm{e}$ \\ Rosemberg Ferreira da Silva ${ }^{3}$
}

Recebido em 13/04/2004. Aceito em 10/09/2004

\begin{abstract}
RESUMO - (Notas sobre a composição arbóreo-arbustiva de uma fisionomia das savanas de Roraima, Amazônia Brasileira). Foi realizado um inventário florístico das espécies arbóreo-arbustivas presentes em uma das unidades de vegetação que compõem a paisagem de savanas do Estado de Roraima, extremo norte da Amazônia brasileira. Esta unidade é caracterizada por ser densamente colonizada por ninhos do cupim Cornitermes ovatus Emerson. Foram observadas 29 espécies (15 famílias botânicas) em três localidades utilizadas para a amostragem. O total de espécies, por localidade, variou de 12 a 20. As espécies mais abundantes foram Byrsonima verbascifolia (L.) DC. e Mimosa microcephala Humb. \& Bonpl. ex Willd. (subarbustivas), Byrsonima cf. intermedia A. Juss. e Randia formosa (Jack.) K. Schum. (arbustivas) e, Byrsonima crassifolia (L.) H.B.K. e Curatella americana L. (arbóreas). Oito espécies são comuns às três localidades. A diversidade medida pelo Índice de Shannon (H') foi baixa para todos os locais amostrados $(<0,90)$, indicando concentração de indivíduos em poucas espécies. O Índice de Sørensen indicou que as áreas estudadas possuem similaridade $( \pm 0,60)$, sugerindo um conjunto de ambientes com diversidade vegetal comum, representando uma mesma unidade de vegetação.
\end{abstract}

Palavras-chave: biodiversidade, Roraima, Amazônia, savana, cerrado, Cornitermes

\begin{abstract}
Notes on the woody composition of a vegetation physionomy of the Roraima's savannas, Brazilian Amazonia). A floristic inventory of woody species was carried out in one of the vegetation units that compose the savannas landscape of the Roraima State, northernmost of Brazilian Amazonia. This unit is characterized by dense colonization of nests of termites Cornitermes ovatus Emerson. Twenty nine woody species were observed (15 botany families) in three localities used for sampling. The total of species varied from 12 to 20 by locality. The most abundant species were Byrsonima verbascifolia (L.) DC. and Mimosa microcephala Humb. \& Bonpl. ex Willd. (dwarf shrubs), Byrsonima cf. intermedia A. Juss. and Randia formosa (Jack.) K. Schum. (shrubby) and, Byrsonima crassifolia (L.) H.B.K. and Curatella americana L. (arboreal). Eight species are common to all localities. Diversity measured by the Index of Shannon $\left(H^{\prime}\right)$ was low for all the areas sampled $(<0.90)$ indicating high specimens concentration in few species. The Index of Sørensen indicated similarities $( \pm 0.60)$ among studied areas, suggesting a group of landscapes with common plant diversity, representing a same vegetation unit.
\end{abstract}

Key words: biodiversity, Roraima, Amazonia, savanna, cerrado, Cornitermes

\section{Introdução}

O maior bloco contínuo de savanas (cerrados) da Amazônia brasileira está situado no complexo paisagístico Rio Branco - Rupununi, distribuído em uma área de $54.000 \mathrm{~km}^{2}$ entre o Brasil e a Guiana (McGill 1966; Eden 1970; Brasil 1975). O lado brasileiro compreende cerca de $39.800 \mathrm{~km}^{2}$ estabelecidos no nordeste do Estado de Roraima (Barbosa \& Fearnside 2005). Toda esta região inclui diversificados ecossistemas que formam um grande mosaico de fisionomias vegetais que podem ser associados a diferentes tipos de solos (Sanaiotti et al. 2002; Miranda et al. 2003) ou grupos animais (Vitt \& Carvalho 1995). Nas áreas centrais destas savanas são identificadas fisionomias onde existem grandes concentrações de ninhos (termiteiros) do cupim Cornitermes ovatus Emerson, criando unidades de vegetação regional com diversidade própria.

Os termiteiros variam de 1-3 m alt., formando agrupamentos em algumas faixas não-contínuas que se estendem do médio curso do rio Cauamé até regiões do alto rio Cotingo, ultrapassando as fronteiras da Guiana. A maior concentração dos ninhos desta espécie

\footnotetext{
1 Instituto Nacional de Pesquisas da Amazônia (Base de Roraima), Rua Coronel Pinto 315, Centro, C. Postal 96, CEP 69301-970, Boa Vista, Roraima, Brasil

2 Convênio INPA/Governo de Roraima, C. Postal 96, CEP 69301-970, Boa Vista, Roraima, Brasil (sabamirr@ technet.com.br)

3 Museu Integrado de Roraima, Av. Brigadeiro Eduardo Gomes s.n., Parque Anauá, CEP 69305-010 Boa Vista, Roraima, Brasil

4 Autor para correspondência: reinaldo@inpa.gov.br
} 
está associada a solos bem drenados, situados na parte mais elevada das leves e médias ondulações do relevo (tesos). Entretanto, eles também podem ser observados em menor densidade em depressões (solos hidromórficos) onde a influencia do nível do lençol freático é visível na estação chuvosa regional, da mesma forma como em algumas localidades denominadas de campos de "murundus" do cerrado do Brasil Central (Araújo Neto et al. 1986; OliveiraFilho 1992).

Como forma de exercício ao conhecimento e compreensão dos ecossistemas regionais, este trabalho teve por objetivo caracterizar a riqueza e a diversidade de espécies da comunidade arbóreo-arbustiva desta fisionomia sob a influência de ninhos de $C$. ovatus, tomando como amostra três diferentes localidades das savanas de Roraima. Este tipo de investigação é importante porque gera informação sob dois caminhos interligados: 1) o da dinâmica e do relacionamento entre os mosaicos de unidades de vegetação que formam a grande paisagem de savanas de Roraima, no sentido de entender a fitogeografia e as determinantes da composição de espécies e diversidade de comunidades, como salientado por Metzger (1999) e 2) gerando parâmetros de análise que possibilitem racionalidade nas políticas públicas de conservação deste ecossistema do extremo norte Amazônico. Toda esta paisagem vem sofrendo rápida degradação devido à ampliação dos cultivos agrícolas de larga escala (soja, arroz e milho) e plantações silviculturais (Acacia mangium Willd. e Eucalyptus spp.), implicando em perda da biodiversidade regional.

\section{Material e métodos}

A atividade de campo consistiu em um inventário florístico rápido da vegetação arbóreo-arbustiva em três áreas de savana sob influência de ninhos de cupins Cornitermes ovatus em Roraima. O levantamento foi entre 25 /novembro e $1 /$ dezembro/2003 e seguiu os seguintes passos:

Seleção das localidades - As áreas onde os termiteiros são encontrados estão sempre situadas em um mosaico das tipologias de savana "gramíneo-lenhosa" (Sg) e "parque" (Sp), ambas definidas pelo IBGE (1992) como ambientes de baixa densidade arbórea (paisagem aberta) dentro das categorias de savanas definidas por aquele órgão. Usando a classificação adotada por Ribeiro \& Walter (1998) para os cerrados do Brasil Central, este ambiente corresponderia a um mosaico de "campo sujo" com "cerrado parque". O tipo de solo onde estão estabelecidos os agrupamentos de termiteiros é, em geral, de alta coesão (latossolo nos tesos e hidromórfico nas depressões), permitindo uma rígida estrutura aos ninhos de $C$. ovatus. Neste sentido, e seguindo a uma prévia análise de campo, foram escolhidas três áreas onde a concentração de ninhos era mais evidente (média estimada de 20 a 30 ninhos.ha ${ }^{-1}$ ), a saber: Fazenda Fortaleza (FFor) localidade próxima da entrada para a Estação Ecológica de Maracá, pela rodovia estadual RR 343 em direção à Vila do Taiano, região do alto curso do rio Cauamé; Aldeia do Contão (ACon) - área próxima da aldeia indígena de mesmo nome, de etnia Macuxi, estabelecida na região do médio curso do rio Cotingo; Fazenda Caracaraizinho (FCar) - região situada entre a margem direita do baixo curso do rio Uraricoera e o lado direito da rodovia BR 174 (direção sul-norte);

Instalação das parcelas amostrais - Após a seleção das áreas, foram montadas três parcelas de $10 \times 100 \mathrm{~m}$ em cada localidade, totalizando 0,9 ha de área amostral total. Este desenho experimental foi concebido porque, em vistorias nas adjacências das parcelas, as espécies arbóreo-arbustivas repetiam-se continuadamente, sendo desnecessário, portanto, a ampliação do número de parcelas amostrais. O ponto inicial de cada parcela foi escolhido aleatoriamente, sendo sempre este a base de seu centro linear. Deste ponto, para traçar o restante da parcela, o rumo a ser seguido foi sempre aquele assumido pela maior concentração dos termiteiros. As coordenadas geográficas (projeção SAD-69) de cada um dos pontos foram as seguintes: FFor-1 (03 $09^{\prime} 56^{\prime \prime} \mathrm{N}$ e $61^{\circ} 09^{\prime} 15^{\prime \prime} \mathrm{W}$ - rumo leste); FFor-2 (03 $100^{\prime} 28^{\prime \prime} \mathrm{N}$ e $61^{\circ} 08^{\prime} 59^{\prime \prime} \mathrm{W}$ - rumo leste); FFor-6 $\left(03^{\circ} 09^{\prime} 55^{\prime \prime} \mathrm{N}\right.$ e $61^{\circ} 09^{\prime} 28^{\prime \prime} \mathrm{W}$ - rumo leste); ACon-3 (04 $09^{\circ} 12^{\prime \prime} \mathrm{N}$ e $60^{\circ} 32^{\prime} 31^{\prime \prime} \mathrm{W}$ - rumo norte); ACon-4 (0409'05" N e 60³2'37' W - rumo norte); ACon-5 (04 $08^{\circ} 36^{\prime \prime}$ " N e $60^{\circ} 32$ '21' W - rumo norte); FCar-7 (03⒉ $7^{\prime} 07^{\prime \prime}$ N e $60^{\circ} 47^{\prime} 46^{\prime \prime} \mathrm{W}$ - rumo norte); FCar-8 (03\%23' $55^{\prime \prime} \mathrm{N} \mathrm{e}$ $60^{\circ} 48^{\prime} 30^{\prime \prime} \mathrm{W}$ - rumo leste); FCar-9 (03 23 ' $11^{\prime \prime}$ N e $60^{\circ} 49^{\prime} 12^{\prime \prime} \mathrm{W}$ - rumo leste);

Inventário florístico (avaliação rápida) - Contagem do número de indivíduos e identificação das espécies arbóreo-arbustivas por unidade de área. Este trabalho foi auxiliado pela divisão de cada parcela em 5 quadras de $10 \times 20 \mathrm{~m}$, no sentido de facilitar a contagem. Espécies não reconhecidas em campo foram codificadas, coletadas e enviadas ao Herbário do Instituto Nacional de Pesquisas da Amazônia (INPA), 
em Manaus, AM, para identificação botânica. O material fértil foi depositado no Herbário do Museu Integrado de Roraima (MIRR);

Caracterização da estrutura vertical da comunidade - Cada indivíduo arbóreo-arbustivo foi reconhecido pelo hábito ( $\mathrm{SB}$ - subarbustivo; $\mathrm{AB}$ arbustivo; AR - árvore) e classificado por categorias de altura $(<1 \mathrm{~m}=$ jovens e formas subarbustivas; entre 1-2 $\mathrm{m}$ = arbóreas em desenvolvimento ou formas arbustivas adultas; $>2 \mathrm{~m}=$ árvores). Esta metodologia baseou-se naquela utilizada por Miranda \& Absy (1997; 2000) para as savanas de Roraima. A altura mínima de entrada no processo de contagem foi de $0,2 \mathrm{~m}$, em virtude da dificuldade de reconhecimento de elementos em fase muito juvenil;

Caracterização da paisagem - Realizada por meio da determinação da densidade (indivíduos por hectare) de cada espécie por categoria de altura, do cálculo da riqueza $(\mathrm{S})$, da diversidade (Shannon - H') e da similaridade florística (Sørensen) entre as três localidades estudadas.

\section{Resultados}

Foram registrados 2.137 indivíduos arbóreoarbustivos por hectare (ind.ha ${ }^{-1}$ ) para a localidade ACon, 1.803 ind.ha $^{-1}$ para FCar e 690 ind.ha $^{-1}$ para FFor, perfazendo uma média de 1.543 ind.ha $^{-1}$ entre as três localidades (Tab. 1). A distribuição média destes indivíduos por categoria estrutural de altura foi de 1.443 $(93,5 \%)$ para indivíduos $<1 \mathrm{~m}, 48(3,1 \%)$ entre $1-2 \mathrm{~m} \mathrm{e}$ $52(3,4 \%)$ para $>2 \mathrm{~m}$. Foi determinado o total de 15 famílias botânicas para 29 espécies nas três áreas, sendo que a maior riqueza foi observada em FCar (20), seguida de ACon (19) e FFor (12).

Duas espécies $(6,9 \%)$ ocuparam exclusivamente o hábito subarbustivo, $14(48,3 \%)$ o arbustivo e 13 $(44,8 \%)$ o arbóreo. As espécies mais abundantes foram Byrsonima verbascifolia e Mimosa microcephala (subarbustivas), Byrsonima cf. intermedia e Randia formosa (arbustivas), e Byrsonima crassifolia e Curatella americana (arbóreas). Estas duas últimas contabilizaram o mesmo número de indivíduos na classe de altura $<1 \mathrm{~m}\left(35,6\right.$ ind.ha $\left.^{-1}\right)$, e valores médios diferentes nas categorias seguintes embora, em ambos os casos, a classe $>2 \mathrm{~m}$ possua um número maior de indivíduos do que a classe de 1-2 m (Fig. 1).

Oito espécies foram comuns às três localidades, sendo que as de maior densidade média foram todas do gênero Byrsonima (Malpighiaceae): B. verbascifolia (subarbustiva), $B$. cf. intermedia (arbustiva) e $B$. crassifolia (arbórea).

O maior índice de diversidade (Shannon - H') foi obtido para a localidade FCar $(0,889)$, seguida de FFor $(0,829)$ e ACon $(0,574)$. A média geral para as três localidades foi de 0,877 . O índice de similaridade de Sørensen variou de 0,56 a 0,62 entre as localidades (Tab. 2).

\section{Discussão}

De forma geral, as três áreas apresentam baixa diversidade árboreo-arbustiva, influenciando diretamente na média geral $\left(\mathrm{H}^{\prime}\right.$ médio $\left.=0,877\right)$, e indicando concentração do número de indivíduos em poucas espécies nesta fisionomia dominada por termiteiros de $C$. ovatus. Resultados de maior amplitude nas savanas de Roraima, obtidos por T.M. Sanaiotti (dados não publicados), Miranda \& Absy (1997; 2000) e Miranda et al. (2003) também descrevem, de forma geral, a baixa diversidade de espécies arbóreo-arbustivas desta região quando comparada a outras áreas do Brasil Central ou mesmo de outras savanas isoladas da Amazônia brasileira. O comum para áreas do cerrado brasileiro (Brasil Central) seria o intervalo de H' situado entre 1,5 e 3,5 (Felfili \& Felfili 2000; Felfili \& Imaña-Encinas 2001). Entretanto, deve-se levar em consideração que a maioria dos estudos fora da região Amazônica representam áreas de cerrados arborizados, ou sensu stricto (naturalmente com maior densidade e diversidade de espécies), com um elevado número de investigações por diferentes localidades. Isto faz, por vezes, pouco produtivo este tipo de comparação com as áreas de menor extensão regional e pouco estudadas do extremo norte amazônico. O trabalho de maior relevância para Roraima foi o de Miranda et al. (2003), que realizou um extenso levantamento amostral de espécies arbóreo-arbustivas por todas as fitofisionomias abertas de savana, relatando um H' de 1,12, pouco acima do determinado como média $(0,877)$ para as áreas de C. ovatus deste estudo.

As localidades estudadas indicam possuir similaridade acima da média, considerando-se os valores obtidos para o índice de Sørensen, sempre próximos a 0,60 . Isto sugere que as três localidades escolhidas representam esta unidade de vegetação regional, não havendo grandes distorções entre seus principais componentes arbóreo-arbustivos. Embora o hábito subarbustivo tenha sido representado por apenas duas espécies (B. verbascifolia e $M$. microcephala) 
Tabela 1. Riqueza, diversidade e densidade (ind.ha ${ }^{-1}$ ) da comunidade arbóreo-arbustiva, por classe de altura e hábito (SB = subarbusto; $\mathrm{AB}=$ arbusto; $\mathrm{AR}=$ árvore), em três localidades das savanas de Roraima (ACon = Aldeia do Contão; FCar = Fazenda Caracaraizinho; FFor $=$ Fazenda Fortaleza)

\begin{tabular}{|c|c|c|c|c|c|c|}
\hline \multirow[t]{2}{*}{ Família/Espécie } & \multirow[t]{2}{*}{ Hábito } & \multirow[t]{2}{*}{ Espécies comuns } & \multicolumn{4}{|c|}{ ACon } \\
\hline & & & $<1 \mathrm{~m}$ & $1-2 \mathrm{~m}$ & $>2 \mathrm{~m}$ & Sub-total \\
\hline \multicolumn{7}{|l|}{ ANNONACEAE } \\
\hline Guatteria sp. & $\mathrm{AR}$ & - & 3,3 & - & - & 3,3 \\
\hline Xylopia aromatica (Lam.) Mart. & $\mathrm{AR}$ & - & - & - & - & - \\
\hline \multicolumn{7}{|l|}{ APOCYNACEAE } \\
\hline $\begin{array}{l}\text { Himatanthus articulatus (Vahl.) Wood. } \\
\text { BIGNONIACEAE }\end{array}$ & AR & $\mathrm{X}$ & - & - & 3,3 & 3,3 \\
\hline Godmania esculifolia H.B.K. Standl. & $\mathrm{AR}$ & - & 36,7 & - & 6,7 & 43,3 \\
\hline \multicolumn{7}{|l|}{ CONNARACEAE } \\
\hline Connarus favosus Planch. & $\mathrm{AB}$ & $\mathrm{X}$ & 160,0 & 13,3 & - & 173,3 \\
\hline $\begin{array}{l}\text { Rourea grosourdyana } \\
\text { var. grosourdyana Baill. }\end{array}$ & $\mathrm{AB}$ & $\mathrm{X}$ & 16,7 & - & - & 16,7 \\
\hline \multicolumn{7}{|l|}{ DILLENIACEAE } \\
\hline Curatella americana $\mathrm{L}$. & AR & $\mathrm{X}$ & 6,7 & - & 10,0 & 16,7 \\
\hline \multicolumn{7}{|l|}{ ERYTHROXILACEAE } \\
\hline Erythroxylum sp. & $\mathrm{AB}$ & - & - & - & - & - \\
\hline Erythroxylum suberosum A. St.-Hill. & $\mathrm{AB}$ & - & - & 3,3 & - & 3,3 \\
\hline \multicolumn{7}{|l|}{ FABACEAE } \\
\hline Bowdichia virgilioides Kunth. & AR & $\mathrm{X}$ & - & 3,3 & - & 3,3 \\
\hline Ormosia sp. & $\mathrm{AR}$ & - & 16,7 & - & - & 16,7 \\
\hline \multicolumn{7}{|l|}{ FLACOURTIACEAE } \\
\hline Casearia sylvestris Swartz & AR & - & 13,3 & - & - & 13,3 \\
\hline \multicolumn{7}{|l|}{ MALPIGHIACEAE } \\
\hline Byrsonima cf. intermedia A. Juss. & $\mathrm{AB}$ & $\mathrm{X}$ & 60,0 & 3,3 & - & 63,3 \\
\hline Byrsonima coccolobifolia Kunth. & AR & & & & & 56,7 \\
\hline Byrsonima crassifolia (L.) H.B.K. & AR & $\mathrm{X}$ & 20,0 & - & - & 20,0 \\
\hline Byrsonima verbascifolia (L.) DC & SB & $X$ & $1.416,7$ & & & $1.416,7$ \\
\hline \multicolumn{7}{|l|}{ MELASTOMATACEAE } \\
\hline Clidemia cf. bullosa DC. & $\mathrm{AB}$ & - & - & - & - & 10,0 \\
\hline Clidemia cf. rubra (Aubl.) Mart. & $\mathrm{AB}$ & - & - & - & - & - \\
\hline Miconia rubiginosa (Bonpl.) DC & $\mathrm{AB}$ & - & - & - & - & - \\
\hline Tibouchina aspera Aubl. & $\mathrm{AB}$ & - & 3,3 & - & - & 3,3 \\
\hline \multicolumn{7}{|l|}{ MIMOSACEAE } \\
\hline Mimosa microcephala Humb. \& Bompl. ex Willd. & SB & - & 106,7 & - & - & 106,7 \\
\hline \multicolumn{7}{|l|}{ MYRTACEAE } \\
\hline Eugenia punicifolia (Kunth.) DC & $\mathrm{AB}$ & - & 3,3 & - & - & 3,3 \\
\hline Eugenia sp. 2 & $\mathrm{AB}$ & - & - & - & - & 6,7 \\
\hline Psidium guianense Pers. & $\mathrm{AB}$ & - & - & - & - & 3,3 \\
\hline \multicolumn{7}{|l|}{ OCHNACEAE } \\
\hline Ouratea sp. & $\mathrm{AR}$ & - & 3,3 & 3,3 & - & 6,7 \\
\hline \multicolumn{7}{|l|}{ PROTEACEAE } \\
\hline Roupala montana Aubl. & $\mathrm{AR}$ & - & - & - & - & 130,0 \\
\hline \multicolumn{7}{|l|}{ RUBIACEAE } \\
\hline Genipa americana $\mathrm{L}$. & AR & - & 3,3 & - & - & 3,3 \\
\hline Palicourea rigida Kunth. & $\mathrm{AB}$ & - & - & & & \\
\hline Randia formosa (Jack.) K. Schum. & $\mathrm{AB}$ & - & 220,0 & - & - & 220,0 \\
\hline Total $\left(\mathrm{n} \cdot \mathrm{ha}^{-1}\right)$ & & - & 2.090 & 27 & 20 & 2.137 \\
\hline Riqueza (S) & & 8 & & & & 19 \\
\hline Índice de Diversidade - Shannon (H') & & - & & & & $\begin{array}{r}0,574 \\
\text { continua }\end{array}$ \\
\hline
\end{tabular}


Tabela 1 (continuação)

Família/Espécie

FCar

FFor

Média

$<1 \mathrm{~m} \quad 1-2 \mathrm{~m} \quad>2 \mathrm{~m}$ Sub-total $<1 \mathrm{~m} \quad 1-2 \mathrm{~m}>2 \mathrm{~m}$ Sub-total $<1 \mathrm{~m} \mathrm{1-2 \textrm {m }}>2 \mathrm{~m}$ Total

\section{ANNONACEAE}

Guatteria sp.

Xylopia aromatica (Lam.) Mart.

APOCYNACEAE

Himatanthus articulatus (Vahl) WoOd. BIGNONIACEAE

Godmania esculifolia H.B.K. Standl.

CONNARACEAE

Connarus favosus Planch.

Rourea grosourdyana var. grosourdyana Baill.

DILLENIACEAE

Curatella americana $\mathrm{L}$.

ERYTHROXILACEAE

Erythroxylum sp.

Erythroxylum suberosum A. St.-Hill.

FABACEAE

Bowdichia virgilioides Kunth.

Ormosia sp.

FLACOURTIACEAE

Casearia sylvestris Swartz

MALPIGHIACEAE

Byrsonima cf. intermedia A. Juss. Byrsonima coccolobifolia Kunth. Byrsonima crassifolia (L.) H.B.K. Byrsonima verbascifolia (L.) DC

MELASTOMATACEAE

Clidemia cf. bullosa DC.

Clidemia cf. rubra (Aubl.) Mart.

Miconia rubiginosa (Bonpl.) DC

Tibouchina aspera Aubl.

MIMOSACEAE

Mimosa microcephala Humb. \& Bompl. ex Willd

MYRTACEAE

Eugenia punicifolia (Kunth.) DC

Eugenia sp. 2

Psidium guianense Pers.

OCHNACEAE

Ouratea sp.

PROTEACEAE

Roupala montana Aubl.

RUBIACEAE

Genipa americana L.

Palicourea rigida Kunth.

Randia formosa (Jack.) K. Schum.

Total (n.ha ${ }^{-1}$ )

Riqueza (S)

Índice de Diversidade - Shannon (H')

$\begin{array}{cccc}- & - & - & - \\ 20,0 & - & 3,3 & 23,3\end{array}$

$-$

106,7

$6,7-$

6,7

$6,7-$

$10,0-$
6,7

10,0

- $\quad-\quad-$

6,7

6,7

3,3

$6,7-$

$43,3 \quad 16,7 \quad 20,0$

80,0

56,7

$\begin{array}{llllllllllllll}- & - & - & & - & & 3,3 & - & - & 3,3 & 1,1 & - & - & \end{array}$

$6,7-6,7$

$-$

$16,7 \quad 3,3 \quad 3,3 \quad 23,3$

$6,7--$

6,7

$$
-
$$$$
-\quad-
$$

$\begin{array}{rrrr}563,3 & 6,7 & - & 570,0\end{array}$

$60,0 \quad-\quad-$

60,0

196,7

$\begin{array}{llll}56,7 & - & 16,7 & 73,3\end{array}$

$30,0 \quad 56,7 \quad 40,0 \quad 126,7$

$476,7 \quad-\quad-\quad 476,7$

$66,7 \quad 10,0 \quad-$

$56,7 \quad-\quad 20,0$

$\begin{array}{lllll}196,7 & 273,3 & 3,3 & - & 276,7\end{array}$

$\begin{array}{rrrrr}76,7 & 41,1 & 3,3 & 5,6 & 50,0\end{array}$

$\begin{array}{lllllllllllllll}10,0 & - & - & 10,0 & - & - & - & - & 3,3 & - & - & & & & \end{array}$

$\begin{array}{rrrrrrrrrrr}- & 3,3 & - & 3,3 & - & - & - & - & - & 1,1 & -\end{array}$

$\begin{array}{llllllllllllll}- & 3,3 & - & 3,3 & & 3 & & - & - & - & & - & 1,1 & -\end{array}$

$\begin{array}{lll}- & 3,3 \\ - & -\end{array}$

$106,7 \quad-\quad-\quad 106,7$

$1,1-$

$35,6 \quad-\quad 35,6$

$\begin{array}{rrrrrrrrrrrr}40,0 & - & - & 40,0 & - & - & - & - & 14,4 & - & - & 14,4 \\ 6,7 & - & - & 6,7 & - & - & - & - & 2,2 & - & - & 2,2 \\ 3,3 & - & - & 3,3 & - & - & - & - & 1,1 & - & - & 1,1\end{array}$

$\begin{array}{llllllllllllll}130,0 & 3,3 & 26,7 & 220,0 & - & - & - & - & & 43,3 & 1,1 & 8,9 & 53,3\end{array}$

$\begin{array}{rrrrrrrrrrrr}- & - & - & - & - & - & - & - & 1,1 & - & - & 1,1 \\ - & - & - & - & 23,3 & - & - & 23,3 & 7,8 & - & - & 7,8 \\ 56,7 & 10,0 & - & 66,7 & - & - & - & - & 92,2 & 3,3 & - & 95,6\end{array}$

$\begin{array}{llllllllllll}1.590 & 103 & 110 & 1.803 & 650 & 13 & 27 & 690 & 1.443 & 48 & 52 & 1.543\end{array}$

$20 \quad 12 \quad 29$

$0,889+0,829$

0,877 


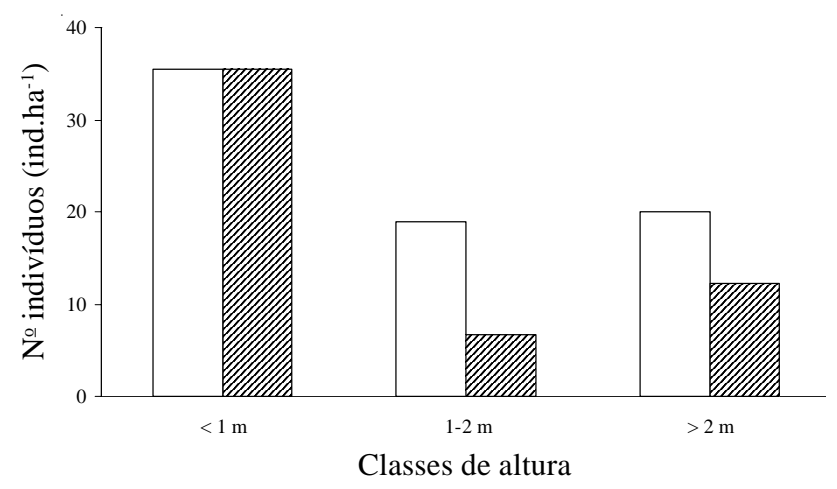

Figura 1. Distribuição do número médio de indivíduos (ind.ha-1) das principais espécies arbóreas, pelas diferentes classes de altura, em três localidades das savanas de Roraima (ACon = Aldeia do Contão; FCar = Fazenda Caracaraizinho; FFor $=$ Fazenda Fortaleza) associadas aos termiteiros de C. ovatus. $\square=$ Byrsonima crassifolia (L.) H.B.K.; $\mathbb{\mathbb { N }}=$ Curatella americana $\mathrm{L}$.

Tabela 2. Índice de Similaridade (Sørensen) entre as três localidades das savanas de Roraima (ACon = Aldeia do Contão; FCar $=$ Fazenda Caracaraizinho $;$ FFor $=$ Fazenda Fortaleza $)$.

\begin{tabular}{lccc}
\hline Localidades & ACon & FCar & FFor \\
\hline ACon & 1 & 0,62 & 0,58 \\
FCar & - & 1 & 0,56 \\
FFor & - & - & 1 \\
\hline
\end{tabular}

no levantamento geral, a densidade de indivíduos de ambas representou altos valores, alcançando 71,3\% de concentração na localidade ACon, dando maior peso a estas espécies e fazendo com que o índice de diversidade fosse o menor dos três $(0,574)$, apesar de possuir 19 das 29 espécies de todo o levantamento. Nesta localidade (ACon), duas das três parcelas que constituíam a amostra, estavam estabelecidas em áreas de contato com solo hidromórfico, limitando a presença das formas arbóreas de espécies como B. crassifolia, B. coccolobifolia e $C$. americana, e incrementando a abundância de $B$. verbascifolia (>1.400 ind.ha $\left.{ }^{-1}\right)$. Nas savanas do Amapá, a maior concentração desta espécie também está relacionada diretamente com o lençol freático (T.M. Sanaiotti, comunicação pessoal).

Das 36 espécies arbóreas (AR) determinadas para todos os tipos de savana de Roraima (Miranda \& Absy 2000), registrou-se 14 distribuídas pelas três localidades estudadas $($ ACon $=11 ;$ FCar $=8$; FFor $=6$ ), sendo que apenas quatro foram encontradas em todas as áreas. Destas, B. crassifolia e C. americana foram as mais representativas, apontando concordância com os principais trabalhos realizados em Roraima (Rodrigues 1958; Takeuchi 1960; Rodrigues 1971;
Dantas \& Rodrigues 1982; Miranda et al. 2003; Barbosa et al. 2003), que indicaram estas duas espécies como as de maior frequiência e abundância do estrato arbóreo das savanas locais. Estas duas espécies também estão fortemente representadas nas savanas do Amapá, sendo usualmente as mais abundantes na maioria das savanas amazônicas (Sanaiotti et al. 1997). Das espécies arbustivas (AB), as mais representativas foram $B$. cf. intermedia e Randia formosa, esta última quase sempre associada a outras espécies, sob a sombra de indivíduos maiores ou aproveitando-se da base de cupinzeiros.

As classes de altura identificadas neste trabalho caracterizam a estrutura vertical da comunidade arbóreo-arbustiva desta unidade de vegetação regional. Há uma grande concentração de indivíduos jovens nas classes de menor tamanho (mesmo excluindo as duas espécies subarbustivas que dominam este estrato), que suplantam em várias vezes as classes de 1-2 m (47,8 \pm

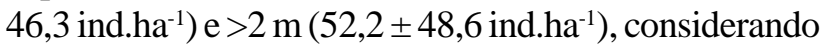
a média das três áreas amostradas. Além de causas naturais como, por exemplo, herbivoria, a forte redução no número de indivíduos que alcançam as classes superiores acontece também por causa da freqüência de fogos que atingem estas áreas no período mais seco do ano (dezembro a março), provocando alta mortalidade nos indivíduos jovens e/ou de baixa resistência (R.I. Barbosa, dados não publicados). Os valores médios de densidade de indivíduos para a classe acima de $2 \mathrm{~m}$ de altura (supracitado), correspondente a espécies com hábito arbóreo neste estudo, são aproximadamente um terço daqueles determinados para "campo sujo" (147 ind.ha ${ }^{-1}$ ) nas savanas de Roraima por Miranda \& Absy (2000).

A fisionomia (unidade de vegetação) caracterizada pela presença de cupinzeiros da espécie $C$. ovatus, na paisagem geral da savana estabelecida no nordeste de Roraima, é caracterizada por apresentar pouca riqueza e baixa diversidade de espécies arbóreo-arbustivas. As principais espécies desta tipologia são $B$. verbascifolia e M. microcephala (subarbustivas), $B$. cf. intermedia e Randia formosa (arbustivas) e B. crassifolia e C. americana (arbóreas). A similaridade entre as localidades estudadas sugere que este ecossistema forma uma tipologia de vegetação comum nas savanas locais. Contudo, diferentes características pedológicas onde os termiteiros estão situados (latossolos não-inundáveis dos "tesos" e solos hidromórficos das suaves depressões) podem provocar variações na abundância de algumas espécies, e devem ser consideradas dentro de estudos de conservação 
ambiental. Estas unidades de vegetação regional são parte da complexidade de mosaicos que compõe a paisagem das savanas de Roraima, possuindo identidade e dinâmica estrutural própria.

\section{Agradecimentos}

Este estudo fez parte dos trabalhos acordados no $3^{\circ}$ Termo Aditivo do Convênio INPA/GERR no $027 / 99$. Os autores agradecem à comunidade indígena da Aldeia do Contão que permitiu o acesso às suas áreas tradicionais; ao Sr. Francisco Araújo Saraiva, proprietário da Fazenda Caracaraizinho e ao Sr. Valdir, capataz da Fazenda Fortaleza, que liberaram suas áreas para a atividade de campo; a Carlos Alberto Cid Ferreira (Herbário INPA), pela colaboração na identificação do material botânico; aos revisores, pela contribuição para a boa qualidade do texto.

\section{Referências bibliográficas}

Araújo Neto, M.D.; Furley, P.A.; Haridasan, M. \& Johnson, C.E. 1986. The murundus of the cerrado region of Central Brazil. Journal of Tropical Ecology 2: 17-35.

Barbosa, R.I. \& Fearnside, P.M. 2005. Fire frequency and area burned in the Roraima savannas of Brazilian Amazonia. Forest Ecology and Management 204: 371-384.

Barbosa, R.I.; Oliveira, A.P.C. \& Lô, D.S. 2003. Caracterização dos ecossistemas terrestres do Projeto de Assentamento Nova Amazônia (PANA). Boa Vista, INPAINCRA, (Relatório de Pesquisa).

Brasil 1975. Projeto RADAMBRASIL. Levantamento de Recursos Naturais, v.8. Rio de Janeiro, Ministério das Minas e Energia.

Dantas, M. \& Rodrigues, I.A. 1982. Estudos fitoecológicos do Trópico Úmido Brasileiro: IV - Levantamentos botânicos em Campos do Rio Branco. Boletim de Pesquisa (EMBRAPA/CPATU) n. 40.

Eden, M. 1970. Savanna vegetation on the northern Rupununi Guyana. The Journal of the Tropical Geography 30: 17-28.

Felfili, J.M. \& Imaña-Encinas, J. 2001. Suficiência de amostragem no cerrado sensu stricto nas quatro áreas estudadas na Chapada do Espigão Mestre do São Francisco. Pp. 31-56. In: J.M. Felfili \& M.C. Silva Júnior (orgs.). Biogeografia do Bioma Cerrado: estudo fitofisionômico na Chapada do Espigão Mestre do São Francisco. Brasília, UnB.
Felfili, M.C. \& Felfili, J.M. 2000. Diversidade alfa e beta no cerrado sensu stricto da Chapada Pratinha, Brasil. Acta Botanica Brasilica 15(2): 243-254.

IBGE 1992. Manual técnico da vegetação brasileira. Manuais Técnicos em Geociências n. 1. Rio de Janeiro, IBGE.

McGill. 1966. Savanna Research Project. MacGill University, Technical Report 5.

Metzger, J.P. 1999. Estrutura da paisagem e fragmentação: análise bibliográfica. Anais da Academia Brasileira de Ciências 71(3-I): 445-463.

Miranda, I.S. \& Absy, M.L. 1997. Flora fanerogâmica das savanas de Roraima. Pp. 445-462. In: R.I. Barbosa; E. Ferreira \& E. Castellón (eds.). Homem, ambiente e ecologia no Estado de Roraima. Manaus, INPA.

Miranda, I.S. \& Absy, M.L. 2000. Fisionomia das savanas de Roraima, Brasil. Acta Amazonica 30(3): 423-440.

Miranda, I.S.; Absy, M.L. \& Rebelo, G.H. 2003. Community structure of woody plants of Roraima savannahs, Brazil. Plant Ecology 164: 109-123.

Oliveira-Filho, A.T. 1992. Floodplain "murundus" of Central Brazil: evidence for termite-origin hypothesis. Journal of Tropical Ecology 8: 1-19.

Ribeiro, J.F. \& Walter, B.M.T. 1998. Fitofisionomias do Bioma Cerrado. Pp. 89-166. In: S.M. Sano \& S.P. Almeida (eds.). Cerrado - Ambiente e Flora. Planaltina - Distrito Federal, EMBRAPA.

Rodrigues, W.A. 1958. Lista dos nomes vernáculos da flora do Território do Rio Branco. Publicação 9. Manaus, INPA, Botânica.

Rodrigues, W.A. 1971. Plantas dos Campos do Rio Branco (Território de Roraima). Pp. 180-193. In: M.G. Ferri (org.). III Simpósio sobre Cerrado. São Paulo, Ed. Edgar Blucher.

Sanaiotti, T.M.; Bridgewater, S. \& Ratter, J.A. 1997. A floristic study of the savanna vegetation of the State of Amapá, Brazil, and suggestions for its conservation. Boletim do Museu Paraense Emílio Goeldi (Série Botânica) 13(1): 3-29.

Sanaiotti, T.M.; Martinelli, L.A.; Victoria, R.L.; Trumbore, S.E. \& Camargo, P.B. 2002. Past vegetation, in Amazon savannas determined using carbon isotopes of soil organic matter. Biotropica 34(1): 2-16.

Takeuchi, M. 1960. A estrutura da vegetação na Amazônia. II . As savanas do norte da Amazônia. Boletim do Museu Paraense Emílio Goeldi (nova série) 7: 1-14.

Vitt, L.J. \& Carvalho, C.M. 1995. Niche partitioning in a tropical wet season: lizards in the Lavrado area of Northern Brazil. Copeia 2: 305-329. 